\title{
POLYNOMIAL IDENTITIES IN GRADED GROUP RINGS, RESTRICTED LIE ALGEBRAS AND $p$-ADIC ANALYTIC GROUPS
}

\begin{abstract}
ANER SHALEV
ABstract. Let $G$ be any finitely generated group, and let $K$ be a field of characteristic $p>0$. It is shown that the graded group $\operatorname{ring} \operatorname{gr}(K G)$ satisfies a nontrivial polynomial identity if and only if the pro- $p$ completion of $G$ is $p$-adic analytic, i.e. can be given the structure of a Lie group over the $p$-adic field $\mathbb{Q}_{p}$.

The proof applies theorems of Lazard, Quillen and Passman, as well as results on Engel Lie algebras and on dimension subgroups in positive characteristic.
\end{abstract}

\section{Main Result}

Let $K$ be a field of characteristic $p>0$, and let $G$ be a finitely generated group. Consider the group ring $K G$, and let $\Delta$ be its augmentation ideal. Put

$$
\operatorname{gr}(K G)=\bigoplus_{n \geq 0} \Delta^{n} / \Delta^{n+1}
$$

viewed as an associative ring. We say that an (associative) ring is a PI ring if it satisfies a nontrivial polynomial identity. Our goal here is to characterize groups $G$ for which $\operatorname{gr}(K G)$ is a PI ring.

Note that group rings satisfying a polynomial identity were characterized by Isaacs and Passman [I-P] (zero characteristic) and subsequently by Passman [P1] (positive characteristic). The main result in characteristic $p$ shows that $K G$ is a PI ring if and only if $G$ has a subgroup $H$ of finite index, whose commutator subgroup $H^{\prime}$ is a finite $p$-group [P1].

Clearly, if $K G$ is a PI ring, then so is $\operatorname{gr}(K G)$, but the converse need not hold. The assumption that $\operatorname{gr}(K G)$ satisfies a (homogeneous multilinear) identity $f\left(x_{1}, \ldots, x_{d}\right)$ merely tells us that

$$
f\left(\Delta^{n_{1}}, \ldots, \Delta^{n_{d}}\right) \leq \Delta^{n_{1}+\cdots+n_{d}+1} \text { for all } n_{1}, \ldots, n_{d} \geq 0 \text {. }
$$

Although we have started with an abstract group $G$, and our question is ringtheoretic in nature, the answer turns out to be formulated in the language of topological groups.

Received by the editors September 21, 1990 and, in revised form, February 21, 1991.

1980 Mathematics Subject Classification (1985 Revision). Primary 16A38, 22E20; Secondary 20F40, 17B35, 16A27. 
A topological group is said to be $p$-adic analytic if it can be given the structure of a Lie group over the $p$-adic field $\mathbb{Q}_{p}$.

Our main result is

Theorem A. Let $G$ be a finitely generated group, and let $K$ be a field of characteristic $p>0$. Then $\operatorname{gr}(K G)$ is a PI ring if and only if the pro-p completion of $G$ is p-adic analytic.

In fact, denoting the pro- $p$ completion of $G$ by $\widehat{G}_{p}$, we only have to assume here that $\widehat{G}_{p}$ is finitely generated (as a topological group).

$p$-adic analytic groups were first studied by Lazard in 1965 [L2], and more recently by A. Lubotzky and A. Mann [L-M1, L-M2]. Following the latter we say that a pro-p group $H$ is powerful, if $H / H^{p}$ is abelian, where $H^{p}$ denotes the closed subgroup generated by the $p$ th powers of $H$ (if $p=2, H^{2}$ should be replaced with $H^{4}$ ).

The basic group-theoretic criterion for analyticity, introduced by Lazard, says that a pro- $p$ group $G$ is $p$-adic analytic if and only if it is finitely generated (as a topological group), and possesses a powerful open subgroup $H$ (necessarily of finite index). By [L-M2] this is equivalent to $G$ being of finite rank, where the rank of a pro- $p$ group is the minimal integer $r$ (possibly infinity) such that every closed (equivalently open) subgroup of $G$ can be generated by $r$ elements. The notions of rank and powerfulness apply to finite $p$-groups, with respect to their discrete topology.

The above-mentioned characterizations of $p$-adic analytic groups, when combined with our theorem, give rise to a more concrete description of the groups $G$ for which $\operatorname{gr}(K G)$ satisfies a nontrivial polynomial identity.

Corollary B. The following are equivalent for a finitely generated group $G$ and a field $K$ of characteristic $p$ :

(1) $\operatorname{gr}(K G)$ is a $P I$ ring.

(2) There exists a constant $s$, such that every epimorphic image $P$ of $G$ which is a finite p-group possesses a powerful subgroup of index at most $p^{s}$.

(3) There exists a constant $r$, such that every epimorphic image $P$ of $G$ which is a finite p-group has rank at most $r$.

This immediately implies

Corollary C. Let $G$ and $K$ be as above, and let $H<G$ be a subgroup of index $p^{n}$ for some $n$. Then $\operatorname{gr}(K G)$ is a PI ring if and only if $\operatorname{gr}(K H)$ is a PI ring.

It is somewhat surprising that this result does not hold for nonfinitely generated groups (see $\S 8$ ).

In the course of the proof we make use of the following criterion for analyticity, which seems to have some independent interest.

Proposition D. Let $G$ be a finitely generated pro-p group, and let $L(G)$ be its associated restricted Lie algebra. Then $G$ is p-adic analytic if and only if $L(G)$ is nilpotent.

For the exact definition of $L(G)$, see $\S 3$.

The above criterion, which stems from Lazard's work [L2], will be given a direct proof. 
The area of $p$-adic analytic groups is currently rather active, and has proved useful in various seemingly unrelated contexts (e.g. linear groups, growth functions of residually finite groups, finite $p$-groups of bounded coclass, etc.). The interested reader is referred to [Lu, L-M3, L-M4, M, M-S, LG, D, S2] and the recent monograph [DDMS].

Our theorem suggests that the highly developed theory of PI rings (see [R] as a general reference) might have some relevance to the study of pro- $p$ groups and $p$-adic analytic groups.

The rest of the paper, which is devoted to a proof of Theorem $\mathrm{A}$, is organized as follows: we begin with an easy reduction to the case of pro- $p$ groups $(\S 2)$; then we apply a theorem of Quillen, presenting $\operatorname{gr}(K G)$ as a universal envelope of a certain restricted Lie algebra $L=L(G)$, constructed from the dimension subgroups of $G(\S 3)$; a characterization of restricted Lie algebras whose enveloping algebras satisfy a polynomial identity, recently given by Passman, is then applied to translate the problem into Lie-algebraic language (§4); some further considerations based on the local nilpotency of $n$-Engel Lie algebras are implemented in order to show that the PI-property of $\operatorname{gr}(K G)$ is equivalent to $L$ being nilpotent $(\S 5)$; in the final step, the nilpotency of $L$ is translated into the language of dimension subgroups; using suitable properties of these subgroups, we ultimately show that $L$ is nilpotent if and only if $G$ is $p$-adic analytic $(\S 6)$, thus completing the proof of the theorem.

A quantitative version of Theorem $A$ is mentioned in $\S 8$. It relates the minimal index of a powerful subgroup in $\widehat{G}_{p}$ (and consequently, the dimension of $\widehat{G}_{p}$ as $p$-adic Lie group) to the degree of the identity satisfied by $\operatorname{gr}(K G)$ and the number of generators of $G$.

In $\S 8$ we also make some preliminary remarks on the nonfinitely generated case, which seems to be of a rather different nature.

Section 7 is devoted to the zero characteristic case, which is considerably easier. We show that, if $\operatorname{char}(K)=0$ and $G$ is any group, then $\operatorname{gr}(K G)$ is a PI ring if and only if $G_{2} / G_{3}$ is a torsion group (in which case $\operatorname{gr}(K G)$ is commutative).

Notation is standard. $\left\{G_{n}\right\}_{n \geq 1}$ stands for the lower central series of $G$, and $G^{n}$ is the subgroup generated by all the $n$th powers (or its closure, if $G$ is a topological group). The (minimal) number of generators of $G$ is denoted by $d(G)$. $\phi(G)$ stands for the Frattini subgroup. Lie products-as well as group commutators-are denoted by [,], and should be interpreted according to the context. Long Lie products and commutators are interpreted using the leftnormed convention.

For a restricted Lie algebra $L$, denote by $x \mapsto x^{[p]}$ its formal $p$ th power operation, and by $u(L)$ its (universal) enveloping algebra. A restricted Lie algebra $L$ is said to be graded (more accurately $\mathbb{N}$-graded) if it can be written as a direct sum of linear spaces $L_{n}(n=1,2, \ldots)$ satisfying $\left[L_{n}, L_{m}\right] \leq L_{n+m}$ and $L_{n}^{[p]} \leq L_{n p}$ for all $n, m$.

\section{REDUCTION TO PRO- $p$ GROUPS}

Let us start with a simple reduction.

Consider the dimension subgroups

$$
D_{n}:=G \cap\left(1+\Delta^{n}\right) \quad(n \geq 1)
$$


studied by Jennings [J1] and Lazard [L1]. It is easy to see that $\operatorname{gr}(K G) \cong$ $\operatorname{gr}(K \widehat{G})$, where $\widehat{G}:=\lim G / D_{n}$. Now, for each $n, G / D_{n}$ is a finite $p$-group. Moreover, the topology induced on $G$ by $\left\{D_{n}\right\}$ coincides with the pro- $p$ topology of $G$. Hence $\widehat{G} \cong \widehat{G}_{p}$-the pro- $p$ completion of $G$, so

$$
\operatorname{gr}(K G) \cong \operatorname{gr}\left(K \widehat{G}_{p}\right) \text {. }
$$

This reduces Theorem A to the case where $G$ is a finitely generated pro- $p$ group. We have to show that, for such $G, \operatorname{gr}(K G)$ is a PI ring if and only if $G$ is $p$-adic analytic.

\section{GRADED GROUP RINGS AND ENVELOPING ALGEBRAS}

Based on results of Jennings and Lazard and on the restricted version of the Poincaré-Birkhoff-Witt theorem, Quillen (1968) was able to present the graded ring $\operatorname{gr}(K G)$ as the enveloping algebra $u(L)$ of a certain restricted Lie algebra $L[\mathrm{Q}]$. In order to describe his result, which plays a fundamental role in this paper, set

$$
L(G):=\bigoplus_{n \geq 1} D_{n} / D_{n+1}
$$

It is well known that the commutation and the $p$ th power operations in $G$ induce on $L(G)$ the structure of a restricted Lie algebra over the field of $p$ elements $F_{p}$ (see [L1]). Moreover, $L(G)$ is graded, in the sense described in $\S 1$.

In order to get a restricted Lie algebra over $K$, define

$$
L_{K}(G):=L(G) \otimes_{F_{p}} K .
$$

Quillen's result may now be formulated.

Quillen's Theorem. $\operatorname{gr}(K G) \cong u\left(L_{K}(G)\right)$.

A similar result holds in the case $\operatorname{char}(K)=0$, except that, in $(5), L(G)$ is viewed as a (nonrestricted) Lie ring, and the tensor product in (6) is defined over the integers. Although the definition of the dimension subgroups $D_{n}$ remains unchanged, their group-theoretic description is substantially different [J2].

As a preliminary application, it is now easy to characterize groups whose graded group algebras are commutative.

Proposition 3.1. For a finitely generated group $G$ and a field $K$ of characteristic $p, \operatorname{gr}(K G)$ is commutative if and only if $\widehat{G}_{p}$ is powerful.

Proof. We may assume that $G$ is a pro- $p$ group. In view of Quillen's Theorem, $\operatorname{gr}(K G)$ is commutative if and only if $L_{K}(G)$ is commutative, which amounts to the commutativity of the restricted Lie algebra $L(G)$. Since $L(G)$ is generated by its first homogeneous component $L_{1}:=G / D_{2}$, this is equivalent to $\left[L_{1}, L_{1}\right]=0$, namely, to $G_{2} \leq D_{3}$. Suppose $p>2$. Then $D_{3}=G_{3} G^{p}$ (see [J1, L1]). Working in $\bar{G}:=G / G^{p}$, we see that $G_{2} \leq D_{3}$ if and only if $\bar{G}_{2}=\bar{G}_{3}$, which is equivalent to $\bar{G}_{2}=1$, namely to $G$ being powerful.

The case $p=2$, which is slightly more involved, is left as an exercise. 
The same argument shows that, for an arbitrary group $G, \operatorname{gr}(K G)$ is commutative if and only if, for all $n, G / D_{n}$ is a powerful $p$-group.

\section{Polynomial identities in enVeloping algebras}

In view of Quillen's Theorem, one would like, at this point, to study restricted Lie algebras $L$ for which $u(L)$ is a PI ring. Luckily enough, this project has been very recently completed by Passmann [P2].

Passman's Theorem. Let $L$ be a restricted Lie algebra over $K$. Then the enveloping algebra $u(L)$ is a PI ring if and only if $L$ possesses restricted subalgebras $A \geq B$ satisfying

(a) $\operatorname{dim}_{K}(L / A)<\infty$ and $\operatorname{dim}_{K}(B)<\infty$.

(b) $B \leq Z(A)$ and $A / B$ is abelian.

(c) The ideal generated by $B$ in $u(B)$ is nilpotent.

We claim that, if we start with a graded restricted Lie algebra $L$, we may require that the subalgebras $A$ and $B$ above be graded as well.

Keeping track of Passman's proof, this will follow from

Lemma 4.1. Let $L=\bigoplus L_{n}$ be a graded Lie algebra, and let $H$ be the subalgebra consisting of all the elements $x \in L$ whose centralizers have finite codimension in $L$. Then $H$ is graded, namely $H=\bigoplus H_{n}$, where $H_{n} \leq L_{n}(n \geq 1)$.

Proof. Let $x \in H$, and suppose $x=\sum_{n=1}^{d} x_{n}$, where $x_{n} \in L_{n}$ and $x_{d} \neq 0$.

We have to show that $x_{n} \in H$ for all $n \leq d$. Arguing by induction on the number of nonzero homogeneous components of $x$, it suffices to prove that $x_{d} \in H$.

Since $L / C_{L}(x)$ is finite dimensional, so is $L_{n} / C_{L_{n}}(x)$ for all $n$. But $C_{L_{n}}(x)$ $=\bigcap_{m} C_{L_{n}}\left(x_{m}\right) \leq C_{L_{n}}\left(x_{d}\right)$. Hence $L_{n} / C_{L_{n}}\left(x_{d}\right)$ is finite dimensional for all $n$.

We claim that, for sufficiently large $n, C_{L_{n}}\left(x_{d}\right)=L_{n}$. In order to show this, let us choose $n_{0}$ with the property that the images of $L_{1}, L_{2}, \ldots, L_{n_{0}}$ in $L / C_{L}(x)$ span $L / C_{L}(x)$ (as a finite-dimensional linear space). Then, for any $n>n_{0}$ and $y_{n} \in L_{n}$, there exist $y_{1}, \ldots, y_{n_{0}}$ in $L_{1}, \ldots, L_{n_{0}}$ respectively, such that the element $y:=y_{n}-y_{n_{0}}-\cdots-y_{1}$ lies in $C_{L}(x)$. This means that $[x, y]=0$ and by a leading term argument $\left[x_{d}, y_{n}\right]=0$. It follows that $C_{L_{n}}\left(x_{d}\right)=L_{n}$ for all $n>n_{0}$.

Finally, $C_{L}\left(x_{d}\right)$ is clearly graded, so $C_{L}\left(x_{d}\right)=\bigoplus C_{L_{n}}\left(x_{d}\right)$. Hence

$$
\operatorname{dim}\left(L / C_{L}\left(x_{d}\right)\right)=\sum_{n=1}^{\infty} \operatorname{dim}\left(L_{n} / C_{L_{n}}\left(x_{d}\right)\right)=\sum_{n=1}^{n_{0}} \operatorname{dim}\left(L_{n} / C_{L_{n}}\left(x_{d}\right)\right)<\infty
$$

so $x_{d} \in H$, as required.

Now, given a graded restricted Lie algebra $L$ such that $u(L)$ is a PI ring, let $H$ be defined as above. It is well known that $H$ is a restricted subalgebra. By [P2, Proposition 5.2] $H$ has finite codimension in $L$. Moreover, $H$ is graded, by the lemma above. Therefore, in order to prove the existence of graded subalgebras $B \leq A \leq L$ satisfying conditions (a)-(c) in Passman's Theorem, we may assume that $L=H$. Lemma 5.3 of [P2], dealing with that case, is easily seen to have a graded version (use the same proof, with $x, y$ homogeneous). 
Applying this version in the proof of [P2, Theorem 6.1], and using the fact that $[A, A]$ is graded whenever $A$ is, we readily get graded restricted subalgebras $A, B$ with the required properties.

In what follows we say that a (graded) restricted Lie algebra $L$ is virtually- $P$ (where $P$ is a property), if $L$ has a (graded) restricted subalgebra of finite codimension satisfying $P$.

Corollary 4.2. If $L$ is virtually-abelian then $u(L)$ is a PI ring; if $u(L)$ is a $P I$ ring, then $L$ is virtually-(nilpotent of class at most two).

\section{ViRTUALly NiLPOTENT LiE ALGEbRAS AND the ENGEL CONDITION}

The properties of being virtually-abelian or virtually-nilpotent, when applied to $L_{K}(G)$, obviously have something to do with the behavior of the dimension subgroups $D_{n}$; but they are not always easy to work with. In this section we overcome this difficulty, by replacing them with a more viable property, namely-nilpotency.

This is made possible by the following result, whose proof relies on the local nilpotency of some Engel Lie algebras. The version given below, which is slightly stronger than what is actually needed here, seems to have some independent interest.

Proposition 5.1. Let $L$ be a resiricted Lie algebra, and let $N$ be a restricted ideal satisfying

(1) $\operatorname{dim}(L / N)<\infty$.

(2) $L^{\left[p^{i}\right]} \leq N$ for some $i$.

(3) $N$ is nilpotent.

Then $L$ is nilpotent.

Proof. Denote the nilpotency class of $N$ by $c$.

Let us first show that $L$ satisfies the $k$-Engel condition

$$
[x, \underbrace{y, \ldots, y}_{k}]=0
$$

for $k=(c+1) p^{i}$. Indeed, by the basic properties of restricted Lie algebras we have

$$
[x, \underbrace{y, \ldots, y}_{k}]=[x, \underbrace{y^{\left[p^{i}\right]}, \ldots, y}_{(c+1)}{ }^{\left[p^{i}\right]}] .
$$

But $y^{\left[p^{i}\right]} \in N$, by condition (2), so that $\left[x, y^{\left[p^{i}\right]}\right] \in N$ as well (recall that $N$ is an ideal). This implies that

$$
[x, \underbrace{y^{\left[p^{i}\right]}, \ldots, y}_{(c+1)}{ }^{\left[p^{i}\right]}] \in[\underbrace{N, N, \ldots, N}_{(c+1)}]=0
$$

which proves that $L$ is $k$-Engel.

We claim that, for some function $f$,

$$
\begin{aligned}
& \text { every } d \text {-generated subalgebra of } L \text { is } \\
& \text { nilpotent of class } \leq f(k, d) .
\end{aligned}
$$


Indeed, in its full generality, this follows from Zelmanov's recent remarkable theorem on the local nilpotency of $k$-Engel Lie algebras. However, in our case a more elementary argument can be given.

Note that the Lie algebra $L / N$ is $p^{i}$-Engel. Since it is finite dimensional, it follows by Engel's Theorem (see, e.g., [B, p. 10]) that $L / N$ is nilpotent. Since $N$ is also nilpotent, $L$ must be soluble. Moreover, its derived length is easily seen to be bounded in terms of $p^{i}$ and $c$.

At this point Gruenberg's Theorem [G] on the local nilpotency of soluble Engel Lie algebras may be applied. Using a suitable relatively free Lie algebra, it is now a standard matter to establish (10).

Now, set $d=\operatorname{dim}(L / N)$ and $b=f(k, c+d)$ (where $f$ is as in (10)). We claim that $L$ is nilpotent of class at most $b$.

In order to show this, choose $x_{1}, \ldots, x_{d} \in L$ whose images form a basis for $L / N$, and let $V$ be the linear subspace of $L$ spanned by $x_{1}, \ldots, x_{d}$. Suppose, by contradiction, that, for some $y_{i} \in L \quad(i=1, \ldots, b+1)$, the Lie product $\left[y_{1}, \ldots, y_{b+1}\right]$ does not vanish. Clearly $L=N+V$, so we may assume that, for each $i$, either $y_{i} \in N$ or $y_{i} \in V$. But $N$ is an ideal and

$$
[\underbrace{N, \ldots, N}_{c+1}]=0 .
$$

Therefore, among $y_{1}, \ldots, y_{b+1}$ there are at most $c$ elements of $N$, say $y_{i_{1}}, \ldots$, $y_{i_{c^{\prime}}}\left(\right.$ where $\left.c^{\prime} \leq c\right)$, and all the rest belong to $V$. It follows that

$$
y_{1}, \ldots, y_{b+1} \in\left\langle y_{i_{1}}, \ldots, y_{i_{c^{\prime}}}, x_{1}, \ldots, x_{d}\right\rangle
$$

where, for a set $X,\langle X\rangle$ denotes the nonrestricted Lie subalgebra generated by $X$.

Now, the subalgebra on the right-hand side of (11) is generated by at most $c+d$ elements, so, by (10), it is nilpotent of class at most $f(k, c+d)=b$. This contradicts our assumption that $\left[y_{1}, \ldots, y_{b+1}\right] \neq 0$, and terminates the proof.

We now apply the proposition in a graded situation.

By a finitely generated restricted Lie algebra we mean a Lie algebra which is finitely generated as a restricted Lie algebra (but not necessarily as an ordinary Lie algebra).

Lemma 5.2. The following are equivalent for a finitely generated graded restricted Lie algebra $L$ :

(1) $L$ is virtually-nilpotent.

(2) $L$ is nilpotent.

(3) $L / Z(L)$ is finite dimensional.

Proof. (1) $\Rightarrow(2)$. Write $L=\bigoplus L_{n}$ and let $M=\bigoplus M_{n}$ be a nilpotent graded restricted subalgebra of finite co-dimension. Then there exists a positive integer $n_{0}$ such that $M_{n}=L_{n}$ for all $n>n_{0}$.

Define $N=\bigoplus_{n>n_{0}} L_{n}$. Clearly, $N$ is a restricted ideal of $L$ which is contained in $M$. Therefore $N$ is nilpotent. Moreover, if $p^{i}>n_{0}$ then $L^{\left[p^{i}\right]} \leq N$. Note also that, since $L$ is finitely generated, every homogeneous component $L_{n}$ is finite dimensional. This implies that $\operatorname{dim}(L / N)<\infty$. Applying Proposition 5.1 we conclude that $L$ is nilpotent. 
(2) $\Rightarrow(3)$. Since $L$ is nilpotent, it is certainly $p^{i}$-Engel for some $i$. This means that $L^{\left[p^{i}\right]}$ is central in $L$, so, for $\bar{L}:=L / Z(L)$ we get $\bar{L}^{\left[p^{i}\right]}=0$. Let $X$ be a (finite) generating set for $\bar{L}$ (as a restricted Lie algebra), and let $L_{0}$ be the nonrestricted subalgebra of $\bar{L}$ generated by $X$. Observe that $L_{0}$ is an ordinary finitely generated nilpotent Lie algebra, so it must be finite dimensional. So let $Y$ be a (finite) basis for $L_{0}$ as a linear space, and put $Y_{j}:=\left\{y^{\left[p^{j}\right]}: y \in Y\right\}$. According to [B, p. 10, Proposition 2], the union of all the $Y_{j}$ 's (where $j=0,1,2, \ldots$ ) spans $\bar{L}$ as a linear space. But $Y_{j}=\{0\}$ for $j \geq i$. Hence $L$ is spanned by the finite set $Y_{0} \cup Y_{1} \cup \cdots \cup Y_{i-1}$, so $\operatorname{dim}(\bar{L})<\infty$.

(3) $\Rightarrow(1)$. Trivial.

Let us now return to the graded restricted Lie algebra $L_{K}(G)$, introduced in $\S 3$.

Note that, since $G$ is finitely generated, so is $L_{K}(G)$. The last equivalences show that, in the case of $L_{K}(G)$, there is no difference between being virtuallyabelian and being virtually-(nilpotent of class at most two), and both properties are equivalent to nilpotency.

It is clear that the nilpotency of $L_{K}(G)=L(G) \otimes K$ does not depend on the underlying field $K$ (as long as $\operatorname{char}(K)=p$ ). Therefore, in view of Corollary 4.2 and Quillen's Theorem, we get

Corollary 5.3. Suppose $G$ is finitely generated. Then $\operatorname{gr}(K G)$ is a PI ring if and only if $L(G)$ is nilpotent.

\section{Dimension SUbGroups}

The missing link in the proof is the following result, which characterizes $p$ adic analytic groups in terms of their associated restricted Lie algebras. Though this essentially stems from Lazard's work [L2], we shall give a complete proof, relying on [L-M1, L-M2] and on some properties of dimension subgroups, discussed in [S1, S-S].

Proposition 6.1. Let $G$ be a finitely generated pro-p group. Then $G$ is p-adic analytic if and only if $L(G)$ is nilpotent.

Proof. Suppose first that $G$ is $p$-adic analytic. Then, by [L-M2, Theorem A], $G$ has finite rank, say $r$. Applying [L-M1, Propositions 1.13 and 4.1.13] it follows that $G$ has an open characteristic subgroup $V$ such that, if $N$ is any open normal subgroup of $G$ which is contained in $V$, then $[V, N] \leq N^{q}$, where $q=p$ if $p>2$ and $q=4$ otherwise. Choose $n_{0}$ with the property that $D_{n_{0}}(G) \leq V$.

Then, for every $m \geq n \geq n_{0}$ we have

$$
\left[D_{n}, D_{m}\right] \leq\left[V, D_{m}\right] \leq\left(D_{m}\right)^{q} \leq D_{q m} \leq D_{2 m+1} \leq D_{n+m+1} .
$$

Therefore, if $L_{n}=D_{n} / D_{n+1}$, then in $L(G)=\bigoplus L_{n}$ we get

$$
\left[L_{n}, L_{m}\right]=0 \text { for all } n, m \geq n_{0}
$$

so $N:=\bigoplus_{n \geq n_{0}} L_{n}$ is an abelian graded restricted ideal. Since $N$ has finite co-dimension, $L(G)$ is virtually-abelian. Applying Lemma 5.2, we conclude that $L(G)$ is nilpotent. 
Suppose now that $L(G)$ is nilpotent, and let us show that $G$ is $p$-adic analytic.

Choose a positive integer $n$, such that

$$
[\underbrace{L, L, \ldots, L}_{n}]=0 \text { and }(p, n)=1 \text {. }
$$

This clearly yields $G_{n} \leq D_{n+1}$ (recall that $\left\{G_{n}\right\}$ denotes the lower central series of $G$ ). According to Lazard's formula (see [L1]), the dimension subgroups in characteristic $p$ are given by

$$
D_{n}=\prod_{j p^{i} \geq n}\left(G_{j}\right)^{p^{i}}
$$

Thus

$$
D_{n+1}=\prod_{j p^{i} \geq n+1}\left(G_{j}\right)^{p^{i}}=G_{n+1} \cdot \prod_{j p^{i} \geq n}\left(G_{j}\right)^{p^{i}} \leq G_{n+1} D_{n}=D_{n}
$$

(for the second equality we have used the fact that $p$ does not divide $n$ ). It follows that

$$
D_{n}=D_{n+1}
$$

which in turn implies analyticity by the appendix of [L2].

Let us give a direct proof for this last implication.

Some consequences of the equality (17) are mentioned in [S1, S-S]. One easy consequence is

$$
D_{m}=D_{m+1} \text { for all } m \geq n \text { which is prime to } p \text { [S1, Lemma 1.7] }
$$

(note that, in our case, (18) may be derived directly, if we replace $n$ with $m$ ). A more significant consequence of the equality $D_{n}=D_{n+1}$ is

$$
\begin{aligned}
\left(D_{m}: D_{m+1}\right) & \leq\left(D_{\ulcorner m / p\urcorner}: D\left\ulcorner_{\ulcorner m / p\urcorner+1}\right)\right. \text { and } \\
D_{m} & =\left(D_{\ulcorner m / p\urcorner}\right)^{p} \quad \text { for all } m \geq n
\end{aligned}
$$

[S-S, Theorem 2.5]; see also [S1, Theorem 1.12].

(Here $\ulcorner x\urcorner$ denotes the smallest integer which is greater than or equal to $x$.)

Choose $i_{0}$ such that $p^{i_{0}} \leq n<p^{i_{0}+1}$, and let us show, using the above results, that the series $\left\{\left(D_{p^{i}}: D_{p^{i+1}}\right)\right\}_{i \geq i_{0}}$ is nonincreasing. Fix $i>i_{0}$ and write

$$
\left(D_{p^{i}}: D_{p^{i+1}}\right)=\prod_{p^{i} \leq m<p^{i+1}}\left(D_{m}: D_{m+1}\right) \text {. }
$$

Observe that, if $p$ does not divide $m$, then by (18), $\left(D_{m}: D_{m+1}\right)=1$, so all these factors may be omitted from the right-hand side of (20). As for the remaining factors, apply (19) to obtain $\left(D_{m}: D_{m+1}\right) \leq\left(D_{m / p}: D_{m / p+1}\right)$. This clearly yields

$$
\left(D_{p^{i}}: D_{p^{i+1}}\right) \leq\left(D_{p^{i-1}}: D_{p^{i}}\right) \quad \text { for all } i>i_{0}
$$

as required.

Set $p^{r}=\left(D_{p^{i_{0}}}: D_{p^{i_{0}+1}}\right)$. Then we have

$$
\left(D_{p^{i}}: D_{p^{i+1}}\right) \leq p^{r} \text { for all } i>i_{0} .
$$


Now, apply the second part of (19) to conclude that, for $i>i_{0}$,

$$
p^{d\left(D_{p^{i}}\right)}=\left(D_{p^{i}}: \phi\left(D_{p^{i}}\right)\right) \leq\left(D_{p^{i}}:\left(D_{p^{i}}\right)^{p}\right)=\left(D_{p^{i}}: D_{p^{i+1}}\right)
$$

(recall that $\phi(H)$ stands for the Frattini subgroup of $H$, which coincides with $H^{\prime} H^{p}$ for pro- $p$ groups $H$ ).

It follows that $d\left(D_{p^{i}}\right) \leq r$ whenever $i>i_{0}$.

Choose $i>i_{0}$ such that $p^{i} \geq r$. Let $D=D_{p^{i}}$ if $p$ is odd, and $D=D_{p^{i+1}}$ otherwise. Then $d(D) \leq p^{i}$. Applying [L-M1, Propositions 1.13 and 4.1.13] for the verbal subgroup $V(G):=D$, it follows that $D$ is powerful. Clearly $(G: D)$ is finite. Therefore $G$ is $p$-adic analytic. This completes the proof of the proposition (and hence of Proposition $D$ ).

Theorem $\mathrm{A}$ is now a direct consequence of Proposition 6.1 and Corollary 5.3.

\section{THE ZERO CHARACTERISTIC CASE}

If the underlying field $K$ has characteristic 0 , the situation is considerably simpler. $L=L_{K}(G)$ is now an ordinary Lie algebra of characteristic 0 , and by a result of Latysev its universal envelope $U(L)$ does not satisfy any nontrivial identity unless $L$ is abelian [La]. But by Quillen's Theorem $\operatorname{gr}(K G) \cong U(L)$. Hence $\operatorname{gr}(K G)$ is a PI ring if and only if $L$ is abelian, which, by the definition of $L$, amounts to $G_{2} \leq D_{3}$.

At this stage we may apply Jennings' formula [J2] for the dimension subgroups $D_{n}$ over $K$, namely $D_{n}=\left\langle x \in G: x^{m} \in G_{n}\right.$ for some $\left.m\right\rangle(n \geq 1)$. It follows that $\operatorname{gr}(K G)$ is a PI ring if and only if $G_{2} / G_{3}$ is a torsion group.

\section{CONCLUDING REMARKS}

It turns out that Theorem $\mathrm{A}$ admits a quantitative version:

If $G$ is a $d$-generated group whose graded group ring (in characteristic $p$ ) satisfies a polynomial identity of degree $n$, then $\widehat{G}_{p}$ possesses a powerful subgroup whose index is bounded above in terms of $p, d$ and $n$; consequently, the rank of $\widehat{G}_{p}$, and its dimension as a $p$-adic Lie group, are bounded in terms of $p, d$ and $n$.

Conversely, if $\widehat{G}_{p}$ has a powerful subgroup of index $i$, and $G$ (or $\widehat{G}_{p}$ ) is $d$ generated, then $\operatorname{gr}(K G)$ satisfies a polynomial identity whose degree is bounded in terms of $d$ and $i$.

The proof combines quantitative versions of the results proved in $\S \S 5$ and 6 with a quantitative version of Passman's Theorem, recently obtained by Petrogradski [Pe].

A final point: While our description of PI graded group rings of characteristic zero is of a global nature, and the results in positive characteristic are strictly local: they do not hold for nonfinitely generated groups.

Indeed, if $G$ is an elementary abelian $p$-group of infinite rank, then $\widehat{G}_{p}$ is not $p$-adic analytic, but $\operatorname{gr}(K G)$ is certainly a PI ring. More elaborate examples of nonfinitely generated $p$-groups indicate that the PI property of $\operatorname{gr}(K G)$ has very little to do with $G$ being powerful-by-finite, finite-by-powerful-by-finite, etc.

Furthermore, it is possible to construct groups $H<G$ with $(G: H)=p$, such that $\operatorname{gr}(K G)$ is a PI ring, while $\operatorname{gr}(K H)$ is not (or vice versa), so even Corollary $\mathrm{C}$ is no longer valid. 
This perplexing situation, which should be dealt with separately, suggests that the solution of the PI problem for graded group rings of positive characteristic may take a rather different form in the case of nonfinitely generated groups.

\section{ACKNOWLEDGMENT}

I am grateful to D. S. Passman for communication of written work, prior to publication.

\section{REFERENCES}

[B] J. A. Bachturin, Lectures on Lie algebras, Akademie-Verlag, Berlin, 1978.

[D] M. P. F. Du Sautoy, Finitely generated groups, p-adic analytic groups, and Poincaré series, Bull. Amer. Math. Soc. (N.S.) 23 (1990), 121-126.

[DDMS] J. D. Dixon, M. P. F. Du Sautoy, A. Mann, and D. Segal, Analytic pro-p groups, London Math. Soc. Lecture Notes Ser., Cambridge Univ. Press, Cambridge, 1991.

[G] K. W. Gruenberg, Two theorems on Engel groups, Proc. Cambridge Philos. Soc. 49 (1953), 377-380.

[I-P] I. M. Isaacs and D. S. Passman, Groups with representations of bounded degree, Canad. J. Math. 16 (1964), 299-309.

[J1] S. A. Jennings, The structure of the group ring of a p-group over a modular field, Trans. Amer. Math. Soc. 50 (1941), 175-185.

[J2] _ The group ring of a class of infinite nilpotent groups, Canad. J. Math. 7 (1955), 169187.

[La] V. N. Latysev, Two remarks on PI algebras, Sibirsk. Math. Z. 4 (1963), 1120-1121. (Russian)

[L1] M. Lazard, Sur les groupes nilpotents et les anneaux de Lie, Ann. Sci. École Norm. Sup. 71 (1954), 101-190.

[L2] _ Groupes analytiques p-adiques, Publ. Math. Inst. Hautes Études Sci. 26 (1965), 389603.

[Lu] A. Lubotzky, A group-theoretic characterization of linear groups, J. Algebra 113 (1988), 207-214.

[LG] C. R. Leedham-Green, Pro-p groups of finite coclass, preprint.

[L-M1] A. Lubotzky and A. Mann, Powerful p-groups I: finite groups, J. Algebra 105 (1987), 484505.

[L-M2] _ Powerful p-groups II: p-adic analytic groups, J. Algebra 105 (1987), 506-515.

[L-M3] _ Residually finite groups of finite rank, Math. Proc. Cambridge Philos. Soc. 106 (1989), 385-388.

[L-M4] __, On groups of polynomial subgroup growth, Invent. Math. 104 (1991), 521-533.

[M] A. Mann, Some applications of powerful p-groups, Groups-St. Andrews 1989.

[M-S] A. Mann and D. Segal, Uniform finiteness conditions in residually finite groups, Proc. London Math. Soc. 61 (1990), 529-545.

[P1] D. S. Passman, Group rings satisfying a polynomial identity, J. Algebra 20 (1972), 103-117.

[P2] __ Enveloping algebras satisfying a polynomial identity, J. Algebra 134 (1990), 469-490.

[Pe] V. M. Petrogradski, Identities in the enveloping algebra for modular Lie superalgebras, J. Algebra 145 (1992), 1-21.

[Q] D. G. Quillen, On the associated graded ring of a group ring, J. Algebra 10 (1968), 411-418.

[R] L. H. Rowen, Polynomial identities in ring theory, Academic Press, New York, 1980.

[S1] A. Shalev, Dimension subgroups, nilpotency indices, and the number of generators of ideals in p-group algebras, J. Algebra 129 (1990), 412-438. 
[S2] _ Characterization of p-adic analytic groups in terms of wreath products, J. Algebra 145 (1992), 204-208.

[S-S] C. M. Scoppola and A. Shalev, Applications of dimension subgroups to the power structure of p-groups, Israel J. Math. 73 (1991), 45-56.

Mathematical Institute, University OF OXford, 24-29 St Giles, Oxford 0X1 3LB, ENGLAND

Current address: Institute of Mathematics, The Hebrew University, Jerusalem 91904, Israel 